\title{
Treatment of an Aedes aegypti colony with the Cry11Aa toxin for 54 generations results in the development of resistance
}

\author{
Gloria Cadavid-Restrepo ${ }^{1,3} /{ }^{+}$, Jorge Sahaza ${ }^{2}$, Sergio Orduz ${ }^{1}$ \\ 'Escuela de Biociencias ${ }^{3}$ Grupo de Microbiodiversidad y Bioprospección, Universidad Nacional de Colombia, Calle 59A 63-20, \\ Medellín, Colombia ${ }^{2}$ Unidad de Biotecnología y Control Biológico, Corporación para Investigaciones Biológicas, Medellín, Colombia
}

To study the potential for the emergence of resistance in Aedes aegypti populations, a wild colony was subjected to selective pressure with CryllAa, one of four endotoxins that compose the Bacillus thuringiensis serovar israelensis toxin. This bacterium is the base component of the most important biopesticide used in the control of mosquitoes worldwide. After 54 generations of selection, significant resistance levels were observed. At the beginning of the selection experiment, the half lethal concentration was $26.3 \mathrm{ng} / \mathrm{mL}$ and had risen to $345.6 \mathrm{ng} / \mathrm{mL}$ by generation 54 . The highest rate of resistance, 13.1, was detected in the 54th generation. Because digestive proteases play a key role in the processing and activation of $\mathrm{B}$. thuringiensis toxin, we analysed the involvement of insect gut proteases in resistance to the Cry $11 \mathrm{Aa}$ B. thuringiensis serovar israelensis toxin. The protease activity from larval gut extracts from the CryllAa resistant population was lower than that of the B. thuringiensis serovar israelensis susceptible colony. We suggest that differences in protoxin proteolysis could contribute to the resistance of this Ae. aegypti colony.

Key words: Bacillus thuringiensis - Aedes aegypti - Cryl1Aa toxin resistance - midgut proteases

Due to the increased resistance of mosquitoes to chemical pesticides and the risks these chemicals pose to humans and the environment, insect control using bacteria, such as Bacillus sphaericus and Bacillus thuringiensis serovar israelensis, has increased in various regions of the world over the last three decades, with a market growth prediction of up to $20 \%$ of the world's pesticide use by 2020 (Whalon \& Wingerd 2003, Kumar et al. 2008). The use of insecticides based on B. sphaericus toxins have achieved moderate commercial success in developed countries, but their high cost discourages their use in many developing countries. Moreover, their long-term use in Culex quinquefasciatus and Culex pipiens control programs underway in France, India, Brazil and China has resulted in resistance in the mosquito populations (Sinègre et al. 1994, Rao et al. 1995, SilvaFilha et al. 1995, Yuan et al. 2000, Nielsen-Leroux et al. 2002). Although the use of insecticides based on $B$. thuringiensis serovar israelensis, which have a broader spectrum of activity against species of Aedes, Culex and Anopheles, has proven to be very effective, their activity in the field is limited because it is affected by various biological and environmental factors and therefore requires frequent application (Mittal 2003). The activity of $B$. thuringiensis serovar israelensis is based in the synergistic effects of Cry and Cyt toxins (Crickmore et al. $1995)$ and reaches half lethal concentration $\left(\mathrm{LC}_{50}\right)$ values between 2-30 $\mathrm{ng} / \mathrm{mL}$, depending on the mosquito species and age of the insects (Orduz et al. 1998, Boujelida et al.

Financial support: COLCIENCIAS (196-2000), UNC

+ Corresponding author: gecadavi@unal.edu.co

Received 14 April 2011

Accepted 10 October 2011
2008). Cry4A, Cry4B and Cryl1A toxins, which range in size from $68-135 \mathrm{kDa}$, contribute to the toxicity of intact crystals in a synergistic manner. The related toxin CytA is haemolytic and cytolytic in vitro and is specifically active against dipteran larvae in vivo (Crickmore 1995).

Despite the potency of the products based on $B$. thuringiensis serovar israelensis, the efficacy of these types of biopesticides has a limitation: the crystals settle to the base of the water column, away from the larval feeding range, within a few days of application. Alternatives have been proposed to overcome this problem, such as the development of live recombinant algae or bacteria that express toxin(s) and remain within the feeding range of mosquito larvae (Romero et al. 2001, Khasdan et al. 2003, Zheng et al. 2007). However, an important concern raised against this approach is the high risk of the development of resistance by the target insects as resistance to individual B. thuringiensis serovar israelensis toxins has been observed in laboratory colonies of $C x$. quinquefasciatus. In one selection experiment in which individual toxins or combinations of toxins from $B$. thuringiensis serovar israelensis were used on laboratory colonies of $C x$. quinquefasciatus, Georghiou and Wirth (1997) observed that lower levels of resistance occurred when all the toxins were used in combination.

The mechanisms of insect resistance to $B$. thuringiensis fall into several categories: altered binding of Cry toxins to receptors in the midgut, alterations in the proteolytic processing of the Cry toxin (decreased rates of activation or increased rates of toxin degradation), elevated immune response or enhanced esterase production (Oppert et al. 1997, Bravo et al. 2007, Bravo \& Soberon 2008) and rapid regeneration of the damaged midgut epithelium, which prevents septicaemia (Ferré \& Van Rie 2002). Recently, Bonin et al. (2009) have shown that the resistance of a natural Ae. aegypti population to B. thuringiensis serovar israelensis toxins was associated with a polymorphism in 
the cadherin gene. In addition to this polymorphism, the under-expression of this gene was observed. Here, our objective was to determine under laboratory conditions the risk of the development of toxin resistance in $A e$. aegypti colonies subjected to the continuous selective pressure of the Cryl1A toxin from B. thuringiensis serovar israelensis. In this paper, we suggest that differences in the proteolytic activity of mosquito larvae midgut enzymes could be responsible for the resistance we observed in this Ae. aegypti colony.

\section{MATERIALS AND METHODS}

Insect strains - To ensure the genetic diversity of the Ae. aegypti colony to be treated with the Cryl1Aa toxin, an artificial colony was established by mixing individuals collected from the urban zones of Medellín (state of Antioquia), Riohacha (state of Guajira), San Andres (state of San Andres) and Valledupar (state of Cesar), Colombia and individuals from the reference standard Rockefeller strain, kindly provided by Dr Gary Clark (Notre Dame University, Indiana, USA). B. thuringiensis-based products had not been previously applied at any of the locations where mosquitoes were collected. Five hundred adults from each location were used to establish the initial colony, which was maintained in quarantine for five generations before the start of the selection experiments. In each of the quarantine generations, between 10,000-15,000 larvae were reared. Prior to the start of the selection experiments, the colony was divided into two groups: one group was kept under continuous selection with Cry11Aa (AaOGr) and the other group was not exposed to selection and was used as a control (AaOGs). Both colonies were maintained in the insectary at $30^{\circ} \mathrm{C}$ with $70-80 \%$ relative humidity and a light:dark photoperiod of 12:12 $\mathrm{h}$. The colonies were reared on an artificial diet under laboratory conditions and located in different rooms that were separated by four doors.

CryllAa toxin solution preparation - The Cryl1Aa toxin used in this study was obtained from the $B$. thuringiensis strain 4Q2-81, carrying the plasmid pHT640 encoding the cryllAa gene (Poncet et al. 1993). Toxin production was performed in a 20-1 volume fermenter under the following conditions: air, $18 \mathrm{lpm}$, temperature, $30^{\circ} \mathrm{C}$, and agitation speed, $300 \mathrm{rpm}$ for $72 \mathrm{~h}$ using a culture medium described by Liu and Bajpai (1995) with modifications $\left(0.5 \mathrm{~g} / \mathrm{L} \mathrm{KH}_{2} \mathrm{PO}_{4}\right.$ and $\left.0.5 \mathrm{~g} / \mathrm{L} \mathrm{K}_{2} \mathrm{HPO}_{4}\right)$. The final whole culture (FWC) was concentrated using a tangential flow filtration system equipped with $0.22 \mu \mathrm{m}$ filter cassettes (Millipore, US).

The spore-crystal suspension was incubated for 30 min with $1 \mathrm{M} \mathrm{NaCl}$ and then washed twice with distilled water. The concentrated FWC was centrifuged, resuspended in phosphate buffered saline (PBS) $(0.24 \mathrm{~g}$ $\mathrm{KH}_{2} \mathrm{PO}_{4}, 1.44 \mathrm{~g} \mathrm{Na}_{2} \mathrm{HPO}_{4}, 8 \mathrm{~g} \mathrm{NaCl}, 0.20 \mathrm{~g} \mathrm{KCl}, 800 \mathrm{~mL}$ $\mathrm{H}_{2} \mathrm{O}$ ) and dried in a mini spray dryer (Büchi) to obtain a final yield of $19.5 \mathrm{~g}$ of spore-crystal technical powder, which was stored at $-20^{\circ} \mathrm{C}$ until use.

Bioassay procedures and selection experiments To determine the baseline susceptibility of the initial AaOG colony to the Cryl1Aa toxin and for larval resistance selection experiments, the technical powder was suspended in PBS and homogenised with glass beads. These suspensions were analysed using the Bradford method to determine protein concentration and crystalspore suspensions were maintained at $4^{\circ} \mathrm{C}$ and used within 10 days (Armengol et al. 2006). Cry11Aa toxin stock suspensions with protein concentrations of 20, 200 and $2,000 \mu \mathrm{g} / \mathrm{mL}$ were generated and bioassays to determine the $\mathrm{LC}_{50}$ were performed in $237 \mathrm{~mL}$ plastic cups containing $100 \mathrm{~mL}$ of tap water and 20 early third instar Ae. aegypti larvae. A minimum of five concentrations were tested in triplicate, with mortality rates ranging from $2-98 \%$. The experiment was performed on three different days and mortality was evaluated after $24 \mathrm{~h}$ of treatment. The results were submitted for probit analysis (Raymond 1995) to determine the $\mathrm{LC}_{50}$. This procedure was performed every third generation on both the Cry11Aa-treated colony (AaOGr) and the untreated colony (AaOGs). To select resistant colonies, 1,000 early third instar larvae were cultured in pans containing $2 \mathrm{~L}$ water combined with the appropriate concentration of Cry11Aa toxin. Mosquito larvae that survived after $24 \mathrm{~h}$ were recovered, rinsed with water, counted and transferred to a clean pan with $2 \mathrm{~L}$ water supplemented with an artificial diet. To obtain at least 1,000 surviving adults to initiate the subsequent generation, selective pressure was placed on 5,000-20,000 larvae per generation. The toxin dosage was adjusted to obtain $75-85 \%$ mortality each generation. Exposure to the Cryl1Aa toxin was applied for 54 consecutive generations. The parallel untreated $\mathrm{Ae}$. aegypti colony, AaOGs, likewise derived from the initial colony, was maintained under the same conditions without Cry11Aa toxin treatment. The adult mosquitoes were placed in cages and fed 10\% sugar water. Blood from adult mice served as food for adult females.

Resistance to the Cryl1Aa toxin was expressed as a resistant ratio (RR), which is the ratio of the $\mathrm{LC}_{50}$ of the selected strain (AaOGr) to that of the unselected strain (AaOGs). For RR1, the $\mathrm{LC}_{50}$ of the AaOGr colony in a given generation was divided by the $\mathrm{LC}_{50}$ of the initial AaOGs generation (Georghiou \& Wirth 1997). For RR2, the $\mathrm{LC}_{50}$ of the AaOGr colony in a given generation was divided by the $\mathrm{LC}_{50}$ of individuals from the AaOGs colony of the same generation.

Aedes aegypti third instar larvae mortality kinetics - To study the mortality kinetics of both Ae. aegypti colonies, 20 early third instar larvae from generation 54 were placed in $100 \mathrm{~mL}$ of dechlorinated water with 500 times the initial $\mathrm{LC}_{50}$ of Cry11Aa $(26.3 \mathrm{ng} / \mathrm{mL})$. Larvae incubated in water with no added CryllAa acted as the negative control. The bioassays were incubated at $30^{\circ} \mathrm{C}$ and mortality was scored every $10 \mathrm{~min}$ for $300 \mathrm{~min}$. The bioassays were performed in triplicate and repeated on three different days (Orduz et al. 1994).

Mosquito larvae midgut protease activity - One hundred $A e$. aegypti third instar larvae from generation 54 from both the AaOGr and AaOGs colonies were washed and placed on ice for $15 \mathrm{~min}$. For each specimen, the gut was excised from the larval body, excluding the peritrophic membrane, immediately submersed in icecold TM buffer (100 mM Tris- $\mathrm{HCl}, \mathrm{pH} 7.2,300 \mathrm{mM}$ mannitol) and homogenised with a syringe fitted with 
a 21-gauge needle. The homogenates were centrifuged at $12,000 \mathrm{rpm}$ for $30 \mathrm{~min}$ at $4^{\circ} \mathrm{C}$ and the supernatants were carefully separated from the pellet. Protein in the supernatant, referred to subsequently as the gut extract, was quantified using the Bradford method and aliquots were stored at $-70^{\circ} \mathrm{C}$ until use (Segura et al. 2000).

To determine the total protease activity, gut extracts from insects of both colonies $(0.5 \mu \mathrm{g}$ of total protein) were subjected to $12 \%$ sodium dodecyl sulphate polyacrylamide gel electrophoresis co-polymerised with $0.1 \%$ gelatin (zymogram analysis) at $4^{\circ} \mathrm{C}$ under reducing conditions without previously boiling the samples, as described by Hames and Rickwood (1990). After electrophoresis, the gels were washed four times with $250 \mathrm{mM}$ Tris- $\mathrm{HCl}, 2 \%$ Triton X-100 buffer ( $\mathrm{pH} \mathrm{7)}$ at $4^{\circ} \mathrm{C}$ and incubated in $50 \mathrm{mM}$ Tris- $\mathrm{HCl}$ buffer $(\mathrm{pH} 7)$ and $50 \mathrm{mM}$ carbonate buffer $\left(\mathrm{NaHCO}_{3}-\mathrm{Na}_{2} \mathrm{HPO}_{4}, \mathrm{pH} 10.3\right)$ for $1 \mathrm{~h}$ at $37^{\circ} \mathrm{C}$. The bands were visualised using Coomassie blue staining. The inhibition of protease activity in the gel was assayed using a mixture of phenylmethylsulfonyl fluoride (PMSF), Np-tosyl-L-lysine chloromethyl ketone (TLCK) and N-ptosyl-L-phenylalanine chloromethyl ketone (TPCK) to a final concentration of $1 \mathrm{mM}$ of each one. All reagents were obtained from Sigma (St. Louis, MO, USA).

To determine the proteolytic activities of gut extract trypsin and chymotrypsin, samples of gut extracts from insects of both colonies were thawed and spun at 12,000 rpm for $2 \mathrm{~min}$ and the supernatants containing soluble gut enzymes were tested using 96-well microplate assays. To determine the trypsin activity, samples containing $50 \mu \mathrm{g} /$ $\mathrm{mL}$ protein were diluted 1:100 in two buffers [buffer $\mathrm{A}(50$ $\mathrm{mM}$ Tris- $\left.\mathrm{HCl}, \mathrm{pH} 7.0,20 \mathrm{mM} \mathrm{CaCl}_{2}\right)$ and buffer $\mathrm{B}(50 \mathrm{mM}$ $\left.\mathrm{NaHCO}_{3}-\mathrm{Na}_{2} \mathrm{CO}_{3}, \mathrm{pH} 10.3\right]$ and $50 \mu \mathrm{L}$ was added to the well of a microplate. To initiate the reaction, $N$ - $\alpha$-benzoylL-arginine $p$-nitroanilide (Sigma) $(100 \mathrm{mg} / \mathrm{mL}$ in dimethyl sulfoxide) was diluted 1:100 in buffer A or B and $50 \mu \mathrm{L}$ was added to each sample well for a final concentration of $1.15 \mathrm{mM}$. After $30 \mathrm{~s}$ incubation at $37^{\circ} \mathrm{C}$, the absorbance was monitored at $405 \mathrm{~nm}$ at $15 \mathrm{~s}$ intervals over a $5 \mathrm{~min}$ period. The change in absorbance per min was calculated using a kinetic module (Biorad 680-XR Microplate reader) and the data were compared for each gut extract. N-succinyl-ala-ala-pro-phe-p-nitroanilide (Sigma) $(100 \mathrm{mg} / \mathrm{mL}$ in dimethyl sulfoxide) was used as a substrate to determine the chymotrypsin activity of the Ae. aegypti midgut and the assay was performed as described above. Each test was performed in triplicate (Oppert et al. 1997).

\section{RESULTS}

Development of Ae. aegypti resistance - The Cry11Aa $\mathrm{LC}_{50}$ of the initial Ae. aegypti colony was $26.3 \mathrm{ng} / \mathrm{mL}$ (22$30 \mathrm{ng} / \mathrm{mL}, 95 \%$ confidence interval). This dose resulted in $15-25 \%$ survival $24 \mathrm{~h}$ after the removal of early fourth instar larvae from the toxin solution and these survivors initiated the first generation of the AaOGr colony.

Tolerance to Cry11Aa began to increase slightly in the AaOGr colony from the 3rd-24th generations (RR1 $=0.8-2.3$, RR2 $=1.0-2.4$ ) and a further small increase in tolerance was observed from the 27 th-36th generation $(\mathrm{RR} 1=2.3-3.2, \mathrm{RR} 2=2.8-3.1)$. Finally, from the 36th54th generations, a steady increase above 5 was observed in the $R R(R R 1=5.6-13.1, R R 2=5.5-10.1)$. These results indicate that, under the conditions used in these experiments, it is possible for Ae. aegypti mosquitoes to develop resistance to the $B$. thuringiensis Cryl1Aa toxin (Table).

Mortality kinetics - Third instar larvae from generation 54 of both AaOG colonies were challenged with 500 times the Cry11Aa $\mathrm{LC}_{50}$ of the initial Ae. aegypti colony $(13.15 \mathrm{mg} / \mathrm{mL})$ to examine additional differences between the Ae. aegypti colonies. Mortality for the AaOGs larvae first occurred after $110 \mathrm{~min}$ (5\%), reaching 50\% after 220 min and $90 \%$ after $300 \mathrm{~min}$, at which point the assay was terminated. On the contrary, mortality for the AaOGr larvae did not exceed 10\% after 300 min (Fig. 1).

Zymogram analysis of mosquito larvae gut extracts - A zymogram analysis was used to assess the relative number of soluble gut proteinases in the extracts from Cryl1Aasusceptible and Cryl1Aa -resistant Ae. aegypti colonies. Three bands were observed between 45-70 kDa with gelatinolytic activity in the gut extracts from both mosquito colonies (Fig. 2). However, the intensity of the AaOGr bands was fainter, suggesting that the protease activity of the gut extracts from this group was lower (Fig. 2, Lane 3). Additionally, the effect of serine protease inhibitors was tested. PMSF, TPCK and TLCK inhibitors used in combination significantly inhibited the protease activity of the gut extracts, suggesting that serine proteases mediate the primary gelatinolytic activity (Fig. 2, Lanes 4, 5).

\section{TABLE}

Variation in the susceptibility of Aedes aegypti larvae subjected to continuous selection pressure with

Bacillus thuringiensis serovar israelensis Cry11Aa toxin for 54 consecutive generations

\begin{tabular}{lcccc}
\hline $\begin{array}{l}\text { Generations } \\
\text { under selection }\end{array}$ & $\mathrm{LC}_{50}$ & $95 \% \mathrm{CI}$ & $\mathrm{RR}^{a}$ & $\mathrm{RR}^{b}$ \\
\hline AaOG & 26.3 & $22.5<\mathrm{LC}<30.1$ & 1.0 & 1.0 \\
AaOGr3 & 31.3 & $27.1<\mathrm{LC}<35.4$ & 1.2 & 1.7 \\
AaOGr6 & 24.9 & $21.5<\mathrm{LC}<28.2$ & 0.9 & 1.8 \\
AaOGr9 & 39.2 & $32.8<\mathrm{LC}<46.6$ & 1.5 & 1.7 \\
AaOGr12 & 34.7 & $30.9<\mathrm{LC}<38.5$ & 1.3 & 2.4 \\
AaOGr15 & 60.1 & $50.6<\mathrm{LC}<71.3$ & 2.3 & 1.7 \\
AaOGr18 & 37.7 & $31.3<\mathrm{LC}<43.0$ & 1.4 & 1.6 \\
AaOGr21 & 28.1 & $22.0<\mathrm{LC}<32.5$ & 1.1 & 1.8 \\
AaOGr24 & 21.0 & $3.0<\mathrm{LC}<33.5$ & 0.8 & 1.6 \\
AaOGr27 & 59.6 & $42.8<\mathrm{LC}<110.3$ & 2.3 & 2.8 \\
AaOGr30 & 68.2 & $58.7<\mathrm{LC}<79.9$ & 2.6 & 2.8 \\
AaOGr33 & 84.6 & $67.3<\mathrm{LC}<127.5$ & 3.2 & 3.1 \\
AaOGr36 & 72.0 & $62.6<\mathrm{LC}<86.0$ & 2.7 & 2.8 \\
AaOGr39 & 162.0 & $133.2<\mathrm{LC}<235.8$ & 6.6 & 5.9 \\
AaOGr42 & 293.88 & $234.9<\mathrm{LC}<410.3$ & 11.1 & 7.5 \\
AaOGr45 & 253.0 & $214.4<\mathrm{LC}<345.8$ & 9.6 & 9.0 \\
AaOGr48 & 296.0 & $251.2<\mathrm{LC}<379.4$ & 11.3 & 9.8 \\
AaOGr51 & 146.4 & $110.1<\mathrm{LC}<237.8$ & 5.6 & 5.5 \\
AaOGr54 & 345.6 & $260.8<\mathrm{LC}<460.23$ & 13.1 & 10.1 \\
\hline
\end{tabular}

$a$ : compared to the first generation before exposure to the Cryl1Aa toxin: $b$ : compared to the contemporary untreated generation; CI: confidence interval; $\mathrm{LC}_{50}$, half lethal concentration; RR: rate of resistance. 
Determination of proteases activities - The trypsin and chymotrypsin activities of the gut extracts of $B$. thuringiensis-susceptible and -resistant Ae. aegypti colonies were compared in buffers of different $\mathrm{pH}$ (7.0 and 10.3). The trypsin activity from susceptible and resistant colonies decreased approximately six and four fold, respectively, at $\mathrm{pH} 7.0$ compared to $\mathrm{pH} 10.3$. Likewise, the chymotrypsin activity of susceptible and resistant colonies decreased nine and six fold, respectively, at $\mathrm{pH} 7.0$ compared to $\mathrm{pH} 10.3$ (Fig. 3). In addition, the relative activities of both midgut serine proteases, trypsin and chymotrypsin, in the Ae aegypti resistant colony, were $28 \%$ and $37 \%$, respectively, of those of the susceptible colony at $\mathrm{pH} 10.3$ (Fig. 3C, D).

\section{DISCUSSION}

In this study, we report that after 54 generations of exposure to the CryllAa toxin of B. thuringiensis serovar israelensis, an Ae. aegypti colony developed significant toxin resistance. The treated colony had a very low mortality rate compared to that of the unselected colony when exposed to 500 times the initial Cry11 $\mathrm{A} \mathrm{LC}_{50}$ toxin, and midgut protease analysis indicated that the activities of trypsin and chymotrypsin in the midguts of insects in the Cryl1A-treated colony had decreased.

The significant resistance of the AaOGr colony to the Cryl1Aa toxin was not observed until generation 39, although a steady increase in tolerance to the Cryl1Aa toxin was observed starting with generation 27. Georghiou and Wirth (1997) generated several colonies of the mosquito $C x$. quinquefasciatus that were resistant to one of several toxins of $B$. thuringiensis serovar israelensis. These colonies reached RRs between 2-42.9 within 28 generations at $\mathrm{LC}_{50}$ and $\mathrm{RRs}$ decreased when a combination of toxins was used $(\mathrm{Cry} 4 \mathrm{~A}+4 \mathrm{~B}+11 \mathrm{~A}+\mathrm{Cyt1} \mathrm{A})$ and increased when the toxin CryllA was used alone. Wirth et al. (2004) used B. thuringiensis subsp. jegathesan to generate a resistant colony of $C x$. quinquefasciatus. The selected colony developed 13 -fold resistance in 22 generations, but this resistance dropped to 2.3 fold by generation 26 and remained low. However, when isolated Cryl1B toxin from this bacterium was used, resistance was detected by generation 18 and reached a maximum of 38 fold that was sustained through generation 40 .

Recently, the persistence of $B$. thuringiensis serovar israelensis spread by humans in a wild mosquito habitat was reported in Switzerland (Tilquin et al. 2008). Furthermore, a resistant colony of Ae. aegypti was generated experimentally following 18 generations of continuous exposure to toxic leaf litter material collected from natural mosquito breeding ponds. When the Ae. Aegypti-resistant colony was challenged with individual Cr4A, Cry4B, Cryl1A and Cyt 1A toxins, the RRs were 30.2, 13.7, 6.3 and 3.0, respectively (Bonin et al. 2009). The exposure of Ae. aegypti larvae to toxins at 500 -fold the $\mathrm{LC}_{50}$ of three mosquitocidal strains of $B$. thuringiensis (israelensis, jegathesan and medellin) caused at least $90 \%$ mortality within 60 min (Orduz et al. 1994). Here, Ae. aegypti larvae from generation 54 that were exposed to 500 times the Cry11 $\mathrm{A} \mathrm{LC}_{50}(13.15$ $\mathrm{mg} / \mathrm{mL}$ ) showed different patterns in mortality kinet-

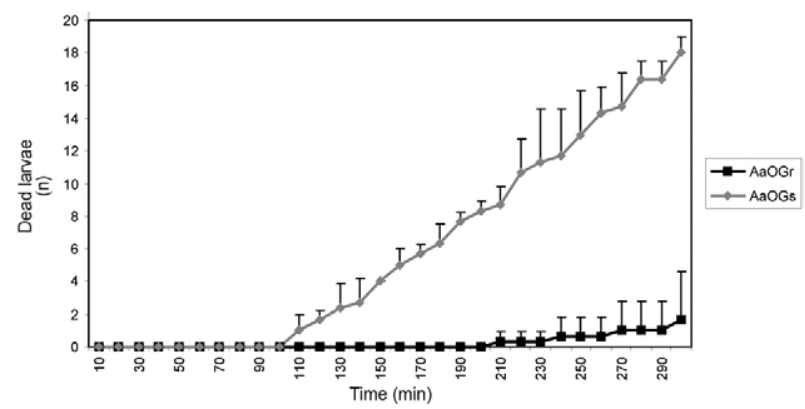

Fig. 1: mortality kinetics of Aedes aegypti third instar larvae from generation 54 selected for resistance to Cry11A toxin compared with the mortality of the contemporary susceptible Ae. aegypti untreated colony. AaOGr: selected strain; AaOGs: unselected strain.

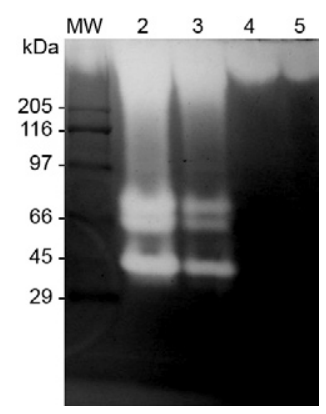

Fig. 2: zymogram analysis of gut extracts of Aedes aegypti colonies. Total protein $(0.5 \mu \mathrm{g})$ was subjected to $12 \%$ sodium dodecyl sulfate polyacrylamide gel electrophoresis co-polymerized with $0.15 \%$ gelatin. Lanes 2, 3 were incubated without mix inhibitors and 4, 5 were incubated in presence to phenylmethylsulfonyl fluoride, N-p-tosyl-Llysine chloromethyl ketone and N-p-tosyl-L-phenylalanine chloromethyl ketone mix inhibitors. Lanes 2, 4: AaOGs colony (unselected strain); 3, 5: AaOGr colony (selected strain); MW: molecular weight.
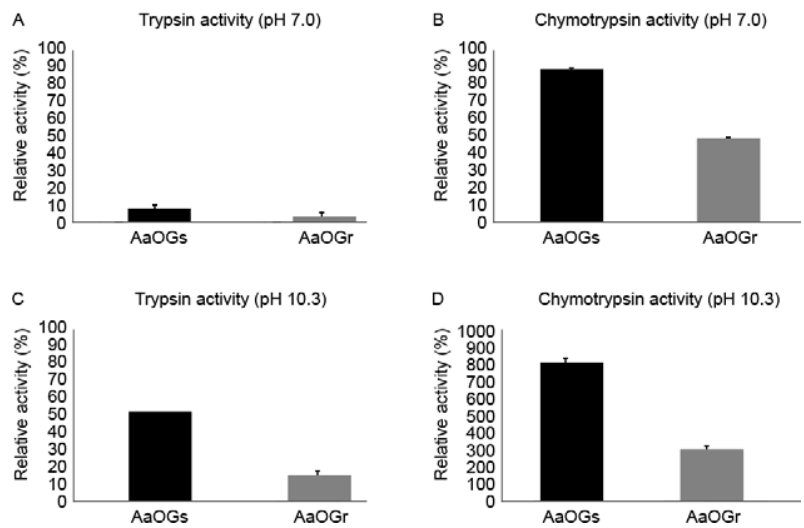

Fig. 3: relative activity of trypsin (A-C) and chymotrypsin (B-D) from Aedes aegypti AaOGs (unselected strain) and AaoGr (selected strain) larvae using the substrates $N$ - $\alpha$-benzoyl-L-arginine $p$-nitroanilide and $\mathrm{N}$-succinyl-ala-ala-pro-phe-p-nitroanilide at $\mathrm{pH} 7.0$ and 10.3, respectively. Bars represent the standard deviation from two independent experiments by triplicate. 
ics. In the AaOGs colony, $90 \%$ of the treated larvae were dead $300 \mathrm{~min}$ after exposure to the Cryl1A toxin; meanwhile, only $10 \%$ of the mosquito larvae from the AaOGr colony were dead in the same time, demonstrating a completely different susceptibility to the Cryl1A toxin. It is possible that the differences in mortality rates following treatment with wild-type strains of B. thuringiensis serovar israelensis (Orduz et al. 1994) compared to treatment with purified Cryl1Aa toxin are due to the synergy that has been reported between the B. thuringiensis serovar israelensis toxins (Tabashnik 1994).

Several instances of resistance to $B$. thuringiensis toxins have been described in Lepidoptera (Plutella xylostella, Plodia interpunctella and Trichoplusia ni), Coleoptera (Leptinotarsa decemlineata and Chrysomela scripta) and Diptera (Cx. quinquefasciatus) species under laboratory, glass house or field conditions, and the strategies of resistance have been determined to include both biochemical and genetic mechanisms (reviewed by Ferré \& Van Rie 2002).

In the case of the $C x$. quinquefasciatus colonies resistant to B. thuringiensis serovar israelensis, Wirth et al. (2010) concluded that the pattern of the inheritance of resistance to the Cryl1Aa toxin was autosomal and that F1 individuals displayed intermediate resistance compared to the parental strains. An evaluation of the genetic diversity of resistant and susceptible Ae. aegypti colonies from Switzerland indicated that there is lower diversity in the resistant colony (Bonin et al. 2008). Further investigations indicated that variations in the genes encoding cadherin and leucine aminopeptidase were responsible for the resistance. In the resistant colony, the cadherin gene encoded a nucleotide polymorphism and its expression was substantially reduced compared to that of the susceptible colony (Bonin et al. 2009).

Trypsin and chymotrypsin-like enzymes are the major digestive proteases of the larval midgut of dipteran insects and are involved in many aspects of the vector - parasite relationship (Ramalho-Ortigao et al. 2003). Moreover, they are critical enzymes in the conversion of $B$. thuringiensis protoxin to toxin. Insects with altered proteinases can evade the effects of $B$. thuringiensis toxins (Ferré \& Van Rie 2002). Additionally, point mutations that provide resistance through the loss of target receptors in midgut membranes have been identified (Darboux et al. 2002). Because each point mutation required to block site-specific toxins would have to occur independently, the probability of developing resistance to $B$. thuringiensis would be proportional to the number of toxins present. The AaOGr colony remained susceptible or had a low tolerance to CryllAa for at least 33 generations of selection, consistent with the observation that Ae. aegypti is the most susceptible mosquito species to $B$. thuringiensis toxins in the Culicidae family (Orduz et al. 1998). The trend of increasing RR values starting with the 24th generation could be evidence of slow change with regard to susceptibility towards CryllAa. Understanding the mechanisms of resistance in vector species, such as $A e$. aegypti, is key to the design of resistance management strategies and to the search for new toxins and strains of $B$. thuringiensis active against mosquitoes.
An important aspect of the mechanism of physiological adaptation and resistance by insects to $B$. thuringiensis toxins is the altered capacity to process protoxin to Cry toxin (Oppert et al. 1997). Variations in the proteolytic processing of the Cry toxin are the result of two distinct routes by which proteinases may affect toxin function, either via decreased rates of activation or increased rates of toxin degradation (Oppert et al. 1997, Bravo \& Soberon 2008). In P. interpunctella and Ostrinia nubilalis, the loss of specific midgut trypsins for converting protoxin to toxin accounted for partial resistance to CrylA toxins (Candas et al. 2003). Resistance in other coleopterans, such as Melolontha melolontha and Leptinotarsa decemlineata, is related to this altered mechanism (Oppert et al. 1997, Karumbaiah et al. 2007). Here, zymogram assays confirmed the presence three bands between $40-70 \mathrm{kDa}$ with protease activity. To verify that the proteins belonged to the serine proteases group, the gel was incubated in presence of inhibitors specific to serine proteases, resulting in the complete depletion of bands. These results suggest that serine proteases are major proteases in the midgut lumen; however the participation of trypsin and chymotrypsin in this process could not be excluded, as they cannot be examined using this approach. Specific substrates were therefore used to determine the activity of these proteases in both colonies. We examined the proteinase activity of the extracts using commercial substrates and determined the protease midgut activity at both neutral $\mathrm{pH}$ (7.0) and at the physiological $\mathrm{pH}$ (10.3) of the mosquito larvae gut because it is known that this parameter is essential to protein solubility and enzymatic activity for toxin efficacy (Segura et al. 2000, Karumbaiah et al. 2007). Using these two $\mathrm{pH}$ conditions, the protease activity of gut extracts was examined for larvae of both $\mathrm{AaOG}$ colonies after the 54th generation, when differences in the mortality kinetics were prominent. The enzymatic activity of the gut extracts was higher at $\mathrm{pH}$ 10.3 than at $\mathrm{pH} 7.0$, consistent with the results of Segura et al. (2000). Additionally, we observed a significant decrease in trypsin and chymotrypsin activity in the resistant colony. These findings corroborate previous reports of proteinase deficiencies in other insect species that have developed resistance to $B$. thuringiensis toxins (Oppert et al. 1997, Candas et al. 2003, Karumbaiah et al. 2007), but the relative contribution of protease alterations to resistance in the AaOGr colony remains to be confirmed, particularly with regard to reports of other mechanisms of resistance. We propose to investigate the susceptibility of the AaOGr colony to other B. thuringiensis toxins, such as Cry4Aa, Cry4Ba, Cry11Ba, $\mathrm{Cry} 11 \mathrm{Bb}$ and $\mathrm{Cyt} 1 \mathrm{~A}$, to perform a proteomic analysis of the midgut proteins of the CryllAa-susceptible and resistant colonies and to determine differences in the binding of CryllAa to midgut proteins between the $\mathrm{AaOGs}$ and AaOGr colonies.

\section{REFERENCES}

Armengol G, Hernandez J, Velez JG, Orduz S 2006. Long-lasting effects of a Bacillus thuringiensis serovar israelensis experimental 
tablet formulation for Aedes aegypti (Diptera: Culicidae) control. $J$ Econ Entomol 99: 1590-1595.

Bonin A, Paris M, Després L, Tetreau G, David JP, Kilian A 2008. A MITE-based genotyping method reveal hundreds of DNA polymorphism in an animal genome after a few generations of artificial selection. BMC Genomics 9: 459.

Bonin A, Paris M, Tetrau G, David J-P, Deprés L 2009. Candidate genes revealed by a genome scan for mosquito resistance to a bacterial insecticide: sequence and gene expression variations. $B M C$ Genomics 10: 551.

Boudjelida H, Aissaoui L, Bouaziz A, Snagghe G, Soltani N 2008. Laboratory evaluation of Bacillus thuringiensis (Vectobac WDG) against mosquito larvae, Culex pipiens and Culiseta longiarelata. Commun Agric Appl Biol Sci 73: 603-609.

Bravo A, Gill SS, Soberon M 2007. Mode of action of Bacillus thuringiensis Cry and Cyt toxins and their potencial for insect control. Toxicon 49: 423-435.

Bravo A, Soberon M 2008. How to cope with insect resistance to Bt toxins? Trends Biotechnol 26: 573-579.

Candas M, Loseva O, Oppert B, Kosaraju P, Bulla LA Jr 2003. Insect resistance to Bacillus thuringiensis: alterations in the indianmeal moth larval gut proteome. Mol Cell Proteomics 2: 19-28.

Crickmore N, Bone EJ, Williams JA, Ellar DJ 1995. Contribution of the individual components of the $\delta$-endotoxin crystal to the mosquitocidal activity of Bacillus thuringiensis subsp. israelensis. FEMS Microbiol Lett 131: 249-254.

Darboux I, Pauchet Y, Castella C, Silva-Filha MH, Nielsen-LeRoux C, Charles JF, Pauron D 2002. Loss of the membrane anchor of the target receptor is a mechanism of bioinsecticide resistance. Proc Natl Acad Sci USA 99: 5830-5835.

Ferré J, Van Rie J 2002. Biochemistry and genetics of insect resistance to Bacillus thuringiensis. Annu Rev Entomol 47: 501-533.

Georghiou G, Wirth MC 1997. Influence of exposure to single versus multiple toxins of Bacillus thuringiensis subsp. israelensis on development of resistance in the mosquito Culex quinquefasciatus (Diptera: Culicidae). Appl Environ Microbiol 63: 1095-1101.

Hames BD, Rickwood D 1990. Gel electrophoresis of proteins: a practical approach, 2nd ed., IRL Press at Oxford University Press, New York, 383 pp.

Karumbaiah L, Oppert B, Jurat-Fuentes JL, Adang MJ 2007. Analysis of midgut proteinases from Bacillus thuringiensis-susceptible and -resistant Heliothis virescens (Lepidoptera: Noctuidae). Comp Biochem Physiol B Biochem Mol Biol 146: 139-146.

Khasdan V, Ben-Dov E, Manasherob R, Boussiba S, Zaritsky A 2003. Mosquito larvicidal activity of transgenic Anabaena PCC 7120 expressing toxin genes from Bacillus thuringiensis subsp. israelensis. FEMS Microbiol Lett 227: 189-195

Kumar S, Chandra A, Pandey KC 2008. Bacillus thuringiensis (Bt) transgenic crop: an environment friendly insect-pest management strategy. J Environ Biol 29: 641-653.

Liu W, Bajpai RK 1995. A modified growth medium for Bacillus thuringiensis. Biotechnol Prog 11: 589-591.

Mittal PK 2003. Biolarvicides in vector control: challenges and prospects. J Vector Borne Dis 40: 20-32.

Nielsen-Leroux C, Pasteur N, Prètre J, Charles J-F, Ben Sheik H, Chevillon C 2002. High resistance to Bacillus sphaericus binary toxin in Culex pipiens (Diptera: Culicidae): the complex situation of West Mediterranean countries. J Med Entomol 39: 729-735.

Oppert B, Kramer KJ, Beeman RW, Johnson D, McGaughey WH 1997. Proteinase-mediated insect resistance to Bacillus thuringiensis toxins. J Biol Chem 272: 23473-23476.
Orduz S, Diaz T, Thiéry I, Charles J-F, Rojas W 1994. Cristal proteins from Bacillus thuringiensis subsp. medellin. Appl Microbiol Biotechnol 40: 794-799.

Orduz S, Realpe M, Arango R, Murillo LA, Delécluse A 1998. Sequence of the cry $11 B b$ gene from Bacillus thuringiensis serovar medellin and toxicity analysis of its encoded protein. Biochem Biophys Acta 1388: 267-272.

Poncet S, Anello G, Delècluse A, Klier A, Rapoport G 1993. Role of the CryIVD polypeptide in the overall toxicity of Bacillus thuringiensis subsp. israelensis. Appl Environ Microbiol 59: 39283930.

Ramalho-Ortigao JM, Kamhawi S, Rowton ED, Ribero JMC, Valenzuela JG 2003. Cloning and characterization of trypsin- and chymotrypsin-like proteases from the midgut of the sand fly vector Phlebotomus papatasi. Insect Biochem Mol Biol 33: 163-171.

Rao DR, Mani TR, Rajendran R, Joseph AS, Gajanana A, Reuben R 1995. Development of high level of resistance to Bacillus sphaericus in a field population of Culex quinquefasciatus from Kochi, India. J Am Mosq Control Assoc 11: 1-5.

Raymond R 1985. Log-probit analysis basic programme of microcomputer. Cahiers ORSTOM series. Ent Med Parasitol 23: 117-121.

Romero M, Gil FM, Orduz S 2001. Expression of mosquito active toxin genes by a Colombian native strain of the gram-negative bacterium Asticcacaulis excentricus. Mem Inst Oswaldo Cruz 96: 257-263.

Segura C, Guzman F, Patarroyo ME, Orduz S 2000. Activation pattern and toxicity of the Cry11Bb1 toxin of Bacillus thuringiensis subsp. medellin. J Invertebr Pathol 76: 56-62.

Silva-Filha MH, Regis L, Nielsen-LeRoux C, Charles J-F 1995. Lowlevel resistance to Bacillus sphaericus in a field-treated population of Culex quinquefasciatus (Diptera: Culicidae). J Econ Entomol 88: 525-530.

Sinègre G, Babinot M, Quermel J-M, Gaven B 1994. First field occurrence of Culex pipiens resistance to Bacillus sphaericus in southern France. In Proceedings of the 8th European Meeting of Society for Vector Ecology, Barcelona, Spain, Society for Vector Ecology, Santa Ana, p. 17.

Tabashnik BE, Finson N, Johnson MW, Heckel DG 1994. Cross-resistance to Bacillus thuringiensis toxin CryIF in the diamondback moth (Plutella xylostella). Appl Environ Microbiol 60: 4627-4629.

Tilquin M, Paris M, Reynaud S, Despres L, Ravanel P, Geremia RA, Gury J 2008. Long lasting persistence of Bacillus thuringiensis subsp. israelensis (Bti) in mosquito natural habitats. PLOS ONE 3: e3432.

Whalon ME, Wingerd BA 2003. Bt: mode of action and use. Arch Insect Biochem Physiol 54: 200-211.

Wirth MC, Delécluse A, Walton WE 2004. Laboratory selection for resistance to Bacillus thuringiensis subsp. jegathesan or a component toxin, Cry11B, in Culex quinquefasciatus (Diptera: Culicidae). J Med Entomol 41: 435-441.

Wirth MC, Walton WE, Federici BA 2010. Inheritance patterns, dominance, stability and allelism of insecticide resistance and crossresistance in two colonies of Culex quinquefasciatus (Diptera: Culicidae) selected with Cry toxins from Bacillus thuringiensis subsp. israelensis. J Med Entomol 47: 814-822.

Yuan Z, Zhang Y, Cai Q, Liu EY 2000. High-level field resistance to Bacillus sphaericus C3-41 in Culex quinquefasciatus from southern China. Biocontrol Sci Tech 10: 41-49.

Zheng D, Valdez-Cruz NA, Armengol G, Sevrez C, Munoz-Olaya JM, Yuan Z, Orduz S, Crickmore N 2007. Co-expression of the mosquitocidal toxins Cyt1Aa and Cryl1Aa from Bacillus thuringiensis subsp. israelensis in Asticcacaulis excentricus. Curr Microbiol 54: 58-62. 